\title{
VERKARSTUNG IM NEUENBURGER JURA
}

\author{
Hans A. Christ
}

Die folgenden Betrachtungen gelten den Karsterscheinungen des Neuenburger Juras, die bisher nicht systematisch untersucht worden sind. Es darf jedoch keine vollzählige Aufzählung aller Formen erwartet werden; insbesondere wird nicht auf die unterirdischen Wasserbahnen eingegangen, die durch die Forschungen von ScHARDT, Rollier, Dubois u. a. gut bekannt sind. Außerdem interessiert in diesem Rahmen nur die landschaftliche Intensität der Karstformen.

Der Neuenburger Jura wird in seinen Hauptzügen durch folgende vier Bergketten bestimmt: die Montagne de Boudry - Chasseral (Seekette), Solmont - Mont Racine - Mont d'Amin, Mont des Verrières - Som Martel und Larmont-Pouillerel. Es sind im wesentlichen kofferartige Antiklinalen, zwischen denen weite Synklinalen (Täler) liegen. Die aufbauenden Gesteine sind überwiegend mesozoisch: Jura und Kreide treten am häufigsten zutage. Sie sind gekennzeichnet durch einen Wechsel von kalkigen und mergeligen Komplexen. Besonders mächtige Kalkserien als möglicher Untergrund von Karsterscheinungen finden sich im SequanKimeridgien (bis über $200 \mathrm{~m}$ Kalke), im Portlandien (über $50 \mathrm{~m}$ ) und im Neocom (Unterkreide, einige Kalkkomplexe über $50 \mathrm{~m}$ ). Die Talböden sind teilweise mit Molasse, teilweise mit glazialen Lehmen und Geschieben belegt, die hydrologisch abdichtend wirken. Areuse und Seyon stellen die wichtigsten Entwässerungslinien dar, die beide nach längerem Synklinallauf im Val de Travers und Val de Ruz die Seekette durchbrechen. Die Mulden von Les Ponts-deMartel, La Brévine und La Chaux-de-Fonds, alle als «la Montagne» zusammengefaßt, liegen wesentlich höher und sind meist unterirdisch entwässert. Im Nordwesten bildet der Doubs streckenweise die Landesgrenze.

Eine hydrographische Abflußrelation läßt sich als allgemeiner Hinweis auf das Maß der oberflächlichen Entwässerung aufstellen. Immerhin sind Kartenungenauigkeiten oder periodische Wasserläufe wesentliche Unsicherheitsfaktoren, so daß sich entsprechende Zahlen von anderen Gebieten nur mit Vorbehalten vergleichen lassen. Für den Neuenburger Jura läßt sich errechnen :

$$
\frac{\text { oberflächliche Wasserläufe in } \mathrm{km}}{\text { Fläche in } \mathrm{km}^{2}}=0,28
$$

(nach der Neuen Landeskarte 1:25 ooo, bezogen auf das Kantonsgebiet). Vergleichszahlen bietet Erzinger (1943, S. 44): Freiberge: 0,31, Ajoie 0,47 und Basler und Aargauer Tafeljura 1,22 .

Karren

\section{DIE KARSTFOR MEN}

Kleine, unregelmäßige, rinnen- oder napfförmige Lösungsformen - sind verschiedentlich zu finden, deutlich in Höhen über $1000 \mathrm{~m}$ NN und bei geneigter Kalklagerung, so an der Tête de Ran und am Pouillerel. Bell (1931, S. 97) beschreibt Karren aus dem Doubstal, wobei er wohl etwas allzu stark die Unterschiede von «lapié de crêts» (Rinnenkarren) bei geneigter Kalklage und «lapié en damier» (Flachkarren) bei horizontalen Kalkbänken betont. Übergänge sind in allen Richtungen vorhanden. MühlethaleR (1931, S. 268) erwähnt karrenähnliche Gebilde aus der Umgebung von Les Verrières, vor allem als «lapiés de crêts», Thiébaud (1937, S.68) aus der Höhenregion der Mtgne. de Boudry und EPPLE (1946, S. 59) vom östlichen Sonnenberg (Val St. Imier). Vegetationsbedeckte Kalkflächen zeigen keine großen und typischen Karrenformen, es sei denn als Reste früherer, unbedeckter Perioden. Chaвот (1927, S. 256) stellt ferner fest, daß alle früher von Gletschern bedeckten Gebiete, so der südliche Jura, viel mehr und deutlichere Karren aufweisen als die unvergletscherten Jura-Plateaux im Nordwesten. Daß eine Vergletscherung als Vorbedingung zur Karrenbildung anzusehen sei, ist indessen verallgemeinert nicht aufrecht zu erhalten. Man denke nur an die aus tropischen Gebieten bekannt gewordenen rezenten Karrenformen. Die Karren sind demnach heute ein weiterer Begriff, morphographisch kaum eindeutig definierbar (vgl. Boegli 1951, S. 203): Es sind genetisch gesprochen 
kleinräumige «subaerische Korrosionsformen», in variablem Zusammenwirken von chemischen und mechanischen Vorgängen (Lösung - Abspülung) entstanden. Im Jura sind Karren wenig entwickelt; sie treten nur kleinräumig auf, so daß nirgends eine landschaftliche Wirkung wie auf «Schrattenfeldern» der helvetischen Kalkalpen (z. B. im Gottesacker-Plateau im Vorarlberg) entsteht. Dagegen spielen

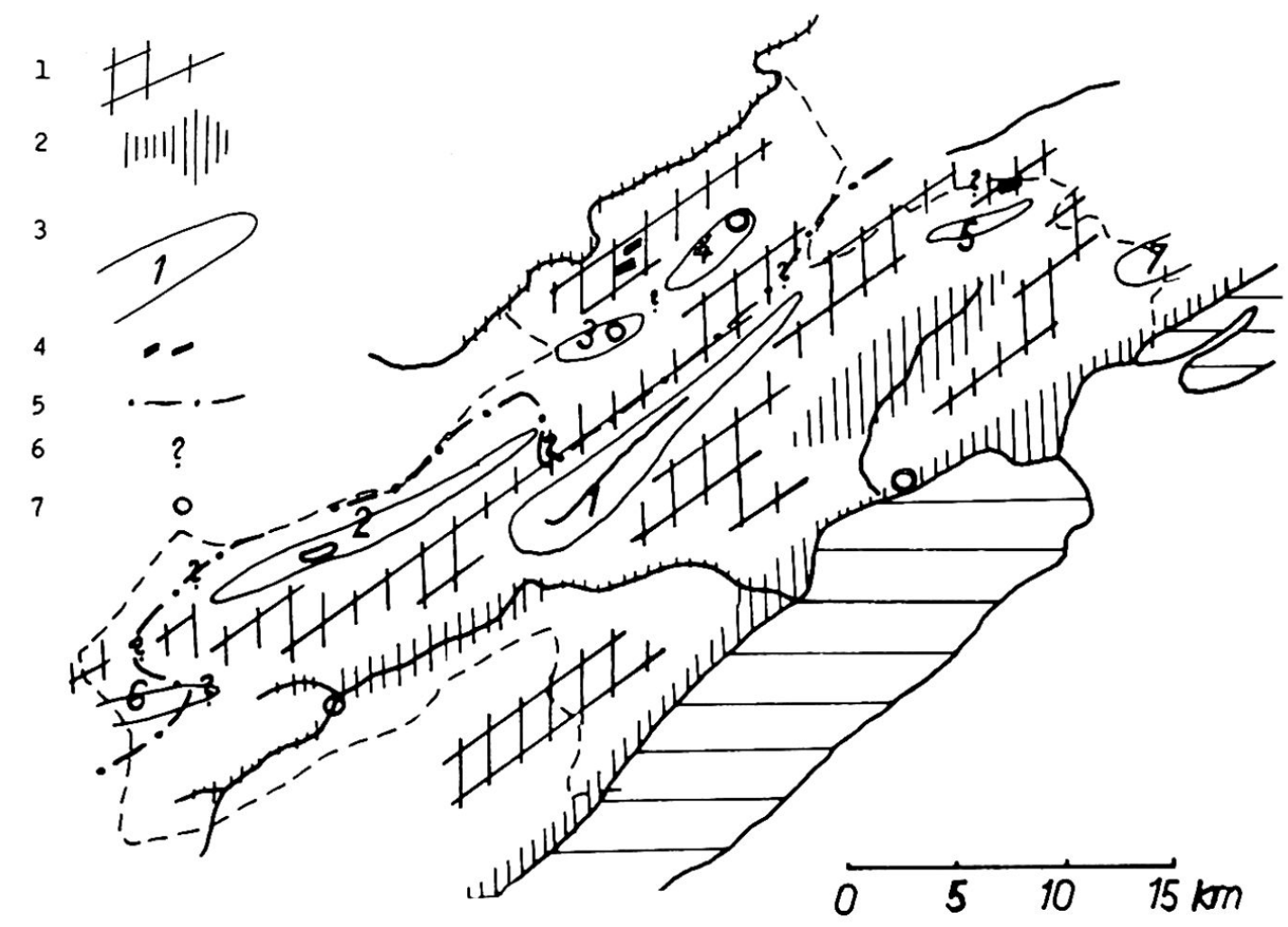

Fig. I Karsthydrographische Skizze. 1 Verkarstete Berggebiete; 2 Oberflächlich entwässerte Gebiete: Vallées, Jurafuß, Doubstal; 3 Geschlossene Becken z. T. vermoort, meist ohne oberflächlichen Abfluß: 1 Becken v. Les Ponts-La Sagne, 2 La Brévine, 3 Le Locle, 4 La Chaux-de-Fonds, 5 Le Coty-Le Pâquier, 6 Les Verrières, 7 Lignières-Tessenberg; 4 Kleine Hochmoore in Antiklinalaufbrüchen; 5 Hauptwasserscheiden Rhône-Rhein; 6 Gebiete mit unsicheren unterirdischen Abflußverhältnissen; 7 Ortschaften

\section{Dolinen}

im weiten Sinne - also trichterähnliche Hohlformen mit rundem, ovalem oder unregelmäßigem Grundriß von sehr wechselnder Größe und Tiefe - eine bedeutende Rolle im Jura, was schon die vielen auch auf unserem Gebiet vorkommenden Lokalnamen: «entonnoirs, emposieux, tannes, gouffres, puits, bétoirs, fondrières» usw. beweisen. Die Formen, die sich unter all den Namen verbergen, sind indessen kaum unter einen genetischen Nenner zusammenzufassen. Ihre Bildung verläuft durchaus nicht überall gleich. Es stehen sich gewissermaßen als Endglieder einer Mischungsreihe die reinen, durch oberflächliche Lösung allmählich schüsselförmig erweiterten Schwunddolinen, also reine Korrosionsformen, und die typischen Einsturzdolinen, die über rasch einbrechenden unterirdischen Hohlräumen oder Spalten entstehen, gegenüber. Sicher sind reine Einsturzdolinen (Erdfälle) viel seltener als die Korrosionsdolinen; oft allerdings mag, gerade im Jura-Gebiet, ein Nachsacken des Bodens mit allmählicher Lösung (vgl. MachatsCheK 1954 und SchäFER 1959) zusammenwirken.

Ob sich auf Grund von Größe, Tiefe und Grundriß einer Doline bereits eine Unterteilung in «junge» und «alte», «unreife» und «reife» Formen (vgl. die Karstzyklus-Theorien!) geben läßt, wie einige Autoren glauben (Снавот, Bell u. a.) ist im Lichte der neueren außereuropäischen Karstforschung zu bezweifeln. Dazu bleibt in unserem Juragebiet die Schwierigkeit, das z. T. sehr enge örtliche Neben- 
einander-Vorkommen der verschiedenartigen Dolinentypen zu erklären. Dolinen treten sehr oft in größerer Zahl auf; meist gilt dann, daß nach äußerem Bild und Entstehung gleichartige Formen beisammen liegen.

Untergrundbedingungen: In einigen Arbeiten über unser Gebiet wird besonders auf die häufigen linearen Dolinenfolgen längs einer Grenze von durchläßigen zu undurchläßigen Schichten hingewiesen; die eigenen Beobachtungen bestätigen dies in vollem Umfang.

Wesentliche Schichtgrenzen solcher Art sind:

Ob. Bathonien/unt. Callovien - Dalle nacrée (z. B. auf der Pouillerel-Antiklinale).

Argovien - «Sequan» (Bes. häufig in typischen Argovien-Comben) (z. B. derrière Tête de Ran, Métairie de Dombresson, Crosettes-Mulde p. p.).

Portlandien - Purbeckien (- ev. Valanginien) z. B. um Bémont in der Vallée de Brévine bei Les Bourquins).

Andererseits erwähnen sowohl Bell (1931, S. 102) als auch Thiébaud (1937, S. 66) Dolinenreihen, die nicht an solche Schichtgrenzen gebunden sind: sie sind nach den erwähnten Autoren auf tektonische Störungszonen zurückzuführen, deren intensive Zerklüftung einen bevorzugten Angriffspunkt für die äußeren Lösungsvorgänge darstellt. Keineswegs also kann man heute mehr mit dem Satz aus MachatscheKs Jurabeschreibung (1905, S. 128) übereinstimmen, $\mathrm{da} \beta$ die Dolinen völlig unabhängig vom geologischen Bau seien.

Orographische Lage: Dolinen finden sich im Talweg, in kausalem Zusammenhang der Tiefenlinie eines Beckens oder Trockentales folgend (z. B. im Trockental bei La Ferrière an der neuenburgisch-bernischen Grenze, oder im Becken «Le Côty», W. von Le Pâquier), sowie auf den breiten Antiklinalrücken oder in den bei höheren Ketten deutlich ausgeprägten Isoklinaltälchen, den Comben. Снавот (1927, S. 257) hält fest, daß Dolinen an Hangpartien immer nur an der Basis, nie am Hang selbst auftreten; dies ist indessen nicht übermäßig zu betonen (wie Erzinger 1943 es tat). Sowohl am Pouillerel und Mont Racine als auch am bernischen Chasseralgrat finden sich gute Beispiele von Dolinen mit teilweise über $10 \mathrm{~m}$ Dm an mehr oder weniger flach geböschtem Hang.

Höhenlage und Häufigkeit: Eine Abhängigkeit von der absoluten Höhe ist allerhöchstens indirekt vorhanden: Die tieferen Talböden sind meist von abdichtenden tertiären oder quartären Sedimenten bedeckt: Dolinen oder Ponore (Schlundlöcher) können sich erst am Kontakt mit Kalken bilden. Sicher sind im Neuenburger Jura in den Höhen von $1000 \mathrm{~m}$ und darüber viel mehr Dolinen zu finden als in den tieferen Tälern der Areuse und des Seyon oder gar am Jura-Südfuß: Dies hängt aber selbstverständlich am starken Relief und der gut ausgebildeten oberflächlichen Entwässerung jener Gegenden.

Form und Ausbildung: Es finden sich alle Übergänge zwischen den flach geböschten, regelmäßigen $W$ annen mit rundem bis ovalem Grundriß und asymmetrischen, kastenähnlichen Senken mit flachem Boden und steilen, oft aus anstehendem Fels gebildeten Rändern. Eigentliche Dolinen haben gemeinsam, daß ihre horizontale Erstreckung wesentlich größer ist als die vertikale (doline, fondrière, entonnoir), im Gegensatz zu den tiefen Schlundlöchern (emposieu, gouffre, puits) von oberflächlich geringen Ausmaßen. Sie kommen in Größen von über $100 \mathrm{~m}$ Dm und über $20 \mathrm{~m}$ Tiefe vor. Die größte Doline unseres Gebietes findet sich mit etwa $200 \mathrm{~m}$ Dm und $30 \mathrm{~m}$ Tiefe auf dem Gewölberücken der Pouillerel-Antiklinale, SE «Maison blanche» (Favre 1910, S. 464). Daneben zeigt aber auf derselben Kette in der Nähe von «Le gros Crêt», südwestlich des Gipfels, eine Schichtgrenzen-Dolinenreihe Formen von nur 1-2 $\mathrm{m}$ Dm und weniger als $1 \mathrm{~m}$ Tiefe. Solche Bergdolinen sind häufig durch Büsche oder Bäume verdeckt, die Taldolinen dagegen oft als freie Mulden erhalten, besonders wenn sie nicht sehr steil geböscht sind, so etwa die Dolinenreihe in der Vallée de Brévine (um Bémont - Les Michel besonders deutlich), oder die großen Dolinen bei «La Clef d'or», ostnordöstlich La Chaux-de-Milieu, oder auch die kleineren Formen im Talboden der Crosettes-Mulde bei «Les Bénéciardes». 


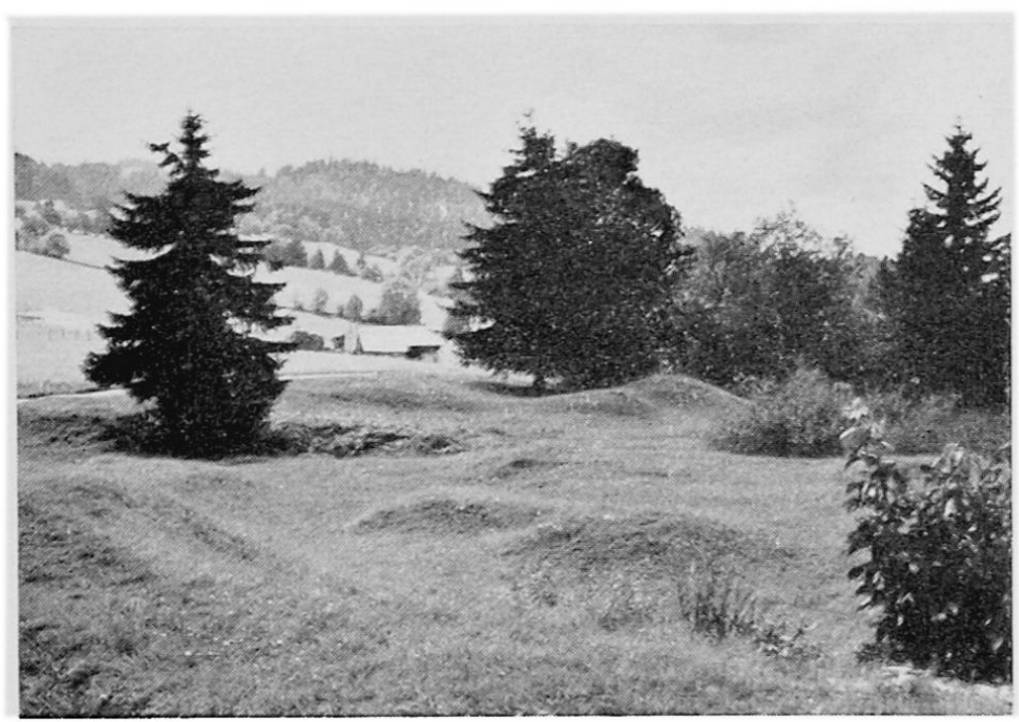

Abb. 1 Bergweide bei a Derrière Pertuis", um $1160 \mathrm{~m}$ hoch. (Mont d'Amin-Antiklinale)

Photo CHRIST

Auch die meist kleineren, aber zahlreich beieinander vorkommenden Dolinen der Argovien-Comben, oft bevorzugte, früh gerodete Bergweiden (z. B. bei «Les Neigeux» derrière Tête de Ran), sind sehr häufig als offene Trichter erhalten, in denen sich bei der dichtenden Mergelunterlage Schmelz- oder Regenwasser einige Zeit halten kann.

Die Schlundlöcher (Ponore) reichen bis in Tiefen von $30-40 \mathrm{~m}$ mit wesentlich geringeren Oberflächenweiten als bei den eigentlichen Dolinen, d. h. sie haben meist übersteile bis senkrechte Abfälle. Daß solche Löcher sehr oft mit unterirdischen Gängen, Spalten oder Höhlen in Verbindung stehen, kann häufig vermutet, ab und zu auch erwiesen werden (vgl. Rickenbach 1925, S. 71, Mühlethaler 1931, S. 272, ThiéBaud 1937, S. 67). Diese Ponore dienen der unterirdischen Entwässerung eines größeren geschlossenen Beckens; sie sind als unzugängliche Löcher meist mit Büschen und Bäumen bestanden und verdeckt. Besonders eindrückliche Beispiele sind die drei großen Emposieux bei «Le Voisinage» (bei Les Ponts-de-Martel), die, an ein Bruchsystem im oberen Malm gebunden (SchardT/Dubois 1902), den teilweise oberflächlich drainierten Ablauf des Beckens von La Sagne - Les Ponts aufnehmen. Das Wasser tritt nach 6-8 Tagen wieder in der Noiraigue-Quelle aus.

In der folgenden Aufstellung finden wir einen von 1) bis 4) zunehmenden landschaftlichen Intensitätsgrad der eigentlichen Dolinen:

1) Reine jurassische Bergweide-Landschaft (Wytweide) ohne Ackerkulturen, mit Büschen oder Bäumen lose bestanden: Die Dolinen sind meist bewaldet und fallen wenig auf. Im Neuenburger Jura finden wir diesen Typ auf den breit abgeflachten Faltenrücken (z. B. in der Südwest-Fortsetzung der Som Martel-Kette zwischen La Brévine und Couvet und in der Gegend um «Derrière Pertuis» auf der Mont d'Amin-Antiklinale (Abb. 1).

2) Jurassische Bergweide-Landschaft in den Isoklinaltälern (Comben), ohne Büsche oder Bäume: Die meist zahlreich beieinander vorkommenden, aber weder sehr großen noch steil geböschten Dolinen treten deutlich hervor. Sie geben der Landschaft ein unruhiges, kraterähnliches Gepräge, ohne indes einen in Kultur oder Besiedlung hervortretenden brüsken Wechsel zu bewirken. (Typische Beispiele bei «Les Neigeux»- derrière Tête de Ran, oder um die Métairie de Dombresson, $\mathrm{N}$ des Chasseral-Grates (Abb. 3).

3) Weide- und Ackerbau-Mischlandschaft, z. Tl. mit Büschen oder Hecken aufgelockert und gegliedert: Dolinen, mehr oder weniger bewachsen, liegen bald in Weiden, bald mit flachen Böschungen in einem Getreidefeld, oder buschbestanden am Ackerrand. Sie lassen die an sich bereits mosaikförmig unregelmäßige Landschaft auch vom Relief her noch unruhiger erscheinen, sie können eventuell sogar Feldgrenzen oder Weglagen beeinflussen. (Das beste Beispiel bietet die von Moränenmaterial gekennzeichnete Umgebung von Les Bayards, nördlich der Talung von Les Verrières; dazu zählt vielleicht noch die Umgebung von Lignières (Tessenberg), die bei stärkerer Drainierung schon einheitlicher, großflächiger kultiviert bzw. beweidet wird.)

4) Weite Synklinal-Talungen, flacher Boden mit Hochmoor-Vegetation oder vereinzelten Ackerbau-Gebieten: Gerade in den Becken von La Brévine und Les Ponts finden sich einige Dolinentrichter von teilweise recht großen Durchmessern ( $50 \mathrm{~m}$ und mehr), die den sonst durchwegs flachen Talboden akzentuieren, und für die Abgrenzung von Ackerfluren oder, wenn sie am Talbodenrand liegen, für die Besiedlungszeile und Weglage bedeutsam werden. Hier 
zeigen sich solche Dolinen am klarsten als Faktoren landschaftlicher Inkohärenz. (Beispiele bei «La Clef d'or» (Abb. 2) oder um La Sagne).

\section{Uvalas, Trockentäler}

Uvalas, d.h. Verschmelzungen von zwei oder mehr Dolinen wurden kleinräumig verschiedentlich beobachtet, sowohl bei Bergdolinen (z. B. um «Derrière Pertuis») als auch bei Taldolinen (z. B.um Bémont); sie bieten aber landschaftlich keine neuen Aspekte.

Bei der Frage der Trockentäler brauchen wir im Jura nicht auf die Uvalas und Dolinen zurückzugreifen: Kein jurassisches Trockental hat sich aus Uvalas, d. h. aus einer allmählich verschmelzenden Dolinenreihe gebildet. In unserem Gebiet ist dies sehr deutlich sowohl bei dem erosiv tief eingeschnittenen, einer großen Transversalstörung folgenden Trockental von Valanvron (bei La Ferrière im neuenburgisch-bernischen Grenzgebiet), das SCHARDT 1911 u. a. untersuchten, als in anderen, mindestens periodischen Trockentälern zu sehen (z. B. Le Chenau, oberhalb des Val de Ruz). Frühere oberirdische Entwässerung und Erosion haben die heutige Form maßgebend bestimmt.

\section{«Bassins fermés》 - 《Poljen》}

Poljen, allseits geschlossene unterirdisch entwässerte Becken sind in der typischen dinarischen Karstlandschaft die wenigen, intensiv bewirtschafteten Ackerbaugebiete, Siedlungs- u. Kulturzentren. Läßt sich dieser Begriff ohne weiteres auf unsere Verkarstungsgebiete übertragen? Die landschaftliche Wirkung unserer rauhen und düsteren, teilweise vermoorten Hochmulden kann kaum gleichgesetzt werden mit derjenigen echter dinarischer Karst-Poljen. Diese äußere Tatsache allein allerdings ist noch kein schlüssiger Beweis gegen den Gebrauch des Begriffes «Polje» für die Juragebiete; sie legt uns indessen doch etwas Vorsicht in der Abgrenzung und Umschreibung einer «Polje»nahe. Viele Detail-Fragen der Poljen-Entstehung sind heute noch umstritten, etwa das Problem der Herkunft der Poljen-Bodenalluvionen, die wohl nicht nur dekalzifizierte Restlehme sein können, oder der progressiven Karstrandebenen-Bildung. Die neuen Untersuchungen von Lou Is (1956) aus dem Taurus zeigen diesbezüglich zum Teil neue Aspekte auf. Machatschek (1905, S. 132 ff.) gebraucht in seiner Jura-Beschreibung den Begriff der Polje gleichwertig mit «bassin fermé». Mit der gegebenen tektonischen Grundlage der Poljen beschreibt er aus dem Kettenjura häufige Muldenpoljen und seltenere Aufbruchspoljen auf Antiklinalen, die aber insgesamt rein erosiv, vor allem durch fließendes Wasser, geformt sind. Ob MachatscheK den etwas überspitzten Satz, daß «zwischen Dolinen und Poljen in der Regel kein genereller Unterschied» vorhanden sei, heute noch so allgemein formulieren würde, sei dahingestellt. Снавот (1927, S. 290 ff.) bleibt bei seiner Studie bei dem für den Jura eingebürgerten Begriff des «bassin fermé»; er identifiziert ihn hydrographisch nicht mit den echten Poljen des dinarischen Gebietes.

Für alle bedeutenden «bassins fermés» des Neuenburger Jura ist charakteristisch: Sie liegen in Synklinalen; die Beckenformen verdanken also ihre Entstehung «in wesentlichem Umfang der Vorarbeit durch gewöhnliche Talbildung unter Verhältnissen, bei denen die Klüfte im Kalk noch nicht in größerem Maße karsthydrographisch (im Sinne von O. LeHManN) wirksam gewesen sind» (Louis 1956, S. 50). Bei JacotGuillarmod (1922) finden wir eine Liste aller «bassins fermés» des Jura, nach Umfang und Abflußverhältnissen abgegrenzt. Vom Gesichtspunkt der landschaftlichen Wirkung bieten kleine und kleinste Formen uns nichts Neues; hier sollen nur noch einige Bemerkungen zu den wichtigen bassins fermés des Neuenburger Juras angefügt werden (vgl. Fig. I).

1) Die Synklinalmulde von Les Ponts - La Sagne, tektonische Fortsetzung der TraversSynklinale, erstreckt sich in SW-NE-Richtung auf etwa $18 \mathrm{~km}$, quer dazu über 0,5 bis über $3 \mathrm{~km}$. Die mittlere Talhöhe liegt knapp über $1000 \mathrm{~m}$, die lokale Erosionsbasis auf etwa $985 \mathrm{~m}$ in 


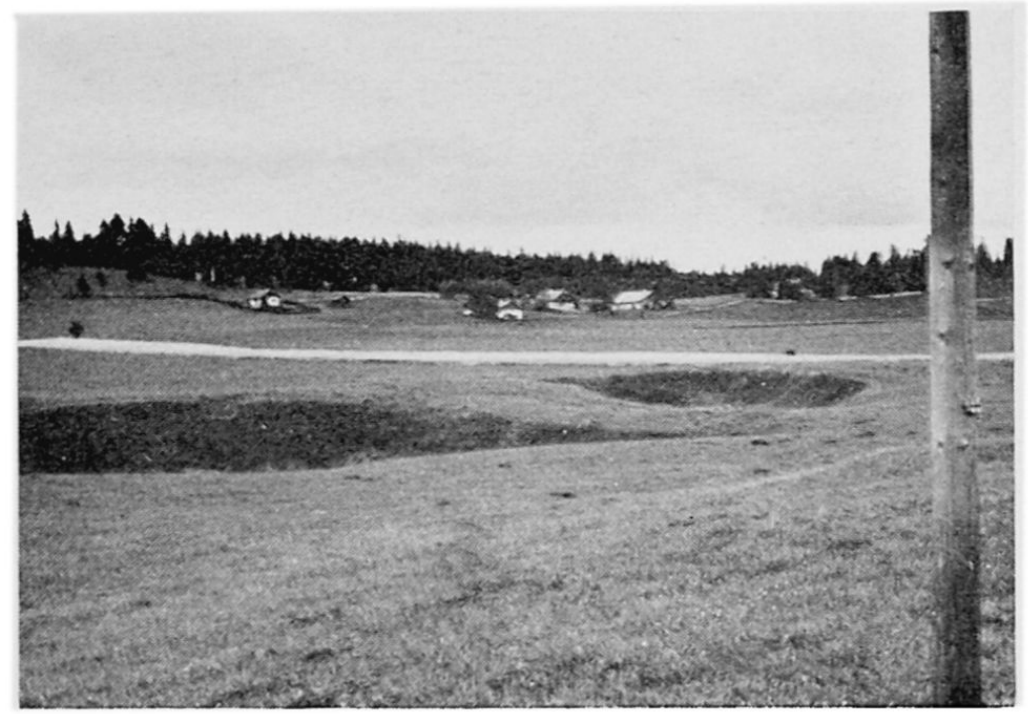

Abb. 2 Taldolinen bei $\propto$ La clef d'or», um $1070 \mathrm{~m}$ hoch, nordwestlich la Chaux-du-Milieu.

Photo Christ

den Ponoren von Le Voisinage. Das versickernde dunkle Moorwasser tritt wieder in der Noiraigue-Quelle (Schwarzwasser!) zu Tage. Der Untergrund von tertiären und quartären Mergeln und Lehmen zusammen mit den klimatischen Verhältnissen ist von entscheidender Bedeutung in diesem «rauhen, kühlfeuchten Hochtal» (GUTERSOHN 1958, S. 76). Auf den bis über $5 \mathrm{~m}$ mächtigen Torfschichten findet sich eine typische Hochmoorvegetation. Der früher in großem Ausmaß betriebene Torfstich wird heute außer lokaler Selbstversorgung nur noch in zwei Betrieben zur Gewinnung von Torfmull-Produkten fortgesetzt. In oberflächlich drainierten Teilen des Beckens ist gegen die Ränder hin ein spärlicher Ackerbau - einige Gerste- und Haferfelder - vorhanden. Typisch sind auch hier die den Beckenrändern entlanglaufenden Siedlungszeilen (La Sagne!): eine Folge der vom Tal streifenförmig zum Berg aneinanderliegenden verschiedenen Nutzungszonen.

2) Die über $20 \mathrm{~km}$ lange Synklinale von La Brévine (vgl. Gutersohn 1950) hat im Gegensatz zum Becken von Les Ponts keinen Talweg mit einheitlichem Gefälle, weist also verschiedene lokale Erosionsbasen auf, so etwa die Versickerungsstelle des Baches von La Brévine oder den unterirdischen $A b f l u B$ des Lac de Taillères, der heute als offene Wasserreserve reguliert ist. Er wurde von Machatschek (1905, S. 138) noch als Poljensee gedeutet, heute wird er eher als ehemaliges Zungenbecken eines Lokalgletschers betrachtet.

3) Den Spezialfall eines bassin fermé bildet das Becken von Lc Locle, dessen oberflächliche Entwässerungsader aus undurchläßigen Tertiärsedimenten zum Kontakt mit den Malmkalken von Col des Roches hinfließt. Dort versickerte der «bied» früher in einem Schlundloch, das indessen größeren Wassermengen nicht gewachsen war: Diese verwandelten es in eine Sumpflandschaft. Seit Beginn des letzten Jahrhunderts (1805) besteht der Durchstich, der das Wasser direkt in einem Kanal durch den Felsen zum tief eingeschnittenen Tal der Rançonnière leitet.

4) Im weiträumig gewellten Gebiet von La Chaux-de-Fonds finden wir bei abklingender Faltungsenergie noch eine generelle Übereinstimmung von Struktur und Orographie (vgl. VOSSELER 1929), wobei aber die Reliefenergie längst nicht mehr so groß ist wie in den vorderen Juraketten. Die Becken der Haupttalung von La Chaux-de-Fonds und der Sekundärsynklinale von Les Crosettes entwässern unterirdisch zum 250-500m tiefer liegenden Lauf des Doubs. Dort entspringen am Talhang zahlreiche Karstquellen, die mit ihren schwankenden Schüttungen ein verspätetes Abbild der Niederschlagsverhältnisse in den Hochmulden zeigen.

5) Die Hochmulde von Le Côty - Le Pâquicr, eine Sekundärstruktur in der nordöstlichen Fortsetzung der breiten Bergschulter des Mont d'Amin, gehört zum peripheren, vorwiegend unterirdisch zur Serrière entwässernden Gürtel rings um das Tal des Seyon. Mit dem Durchbruch von «Pertuis», der die orographische Hauptkammlinie nach N verschiebt, fällt der Abfluß der beiden $N$ anschließenden bewaldeten Comben auch in dieses Becken, wo er in der Nähe von «Le Sauvage» verschwindet.

6-7) Die rauhe Hochmulde von Les Verrières (um 930 m NN) und das Plateau-Becken von Lignières-Tessenberg zeigen beide reichlich unbestimmte Entwässerung. Ober- und unterirdische Abflüsse treten zugleich auf, ihre genaue Abgrenzung fällt schwer. Der träge MorteLauf der Synklinale von Les Verrières fließt zum Doubs, aber unterirdische Abflüsse gehen zur Areuse-Quelle. Die mit glazialen Deponien bedeckte Tessenbergterrasse war in ihrem innersten Teil vermoort, was auch aus der typischen Randlage der Dorfsiedlungen herauszulesen ist. Durch Meliorierungen und Drainierungen wird heute der größte Teil oberflächlich entwässert, 
Abb. 3 Combendolinen um Métairie de Dombresson, um 1400 m hoch, Chasseral Photo CHRIST

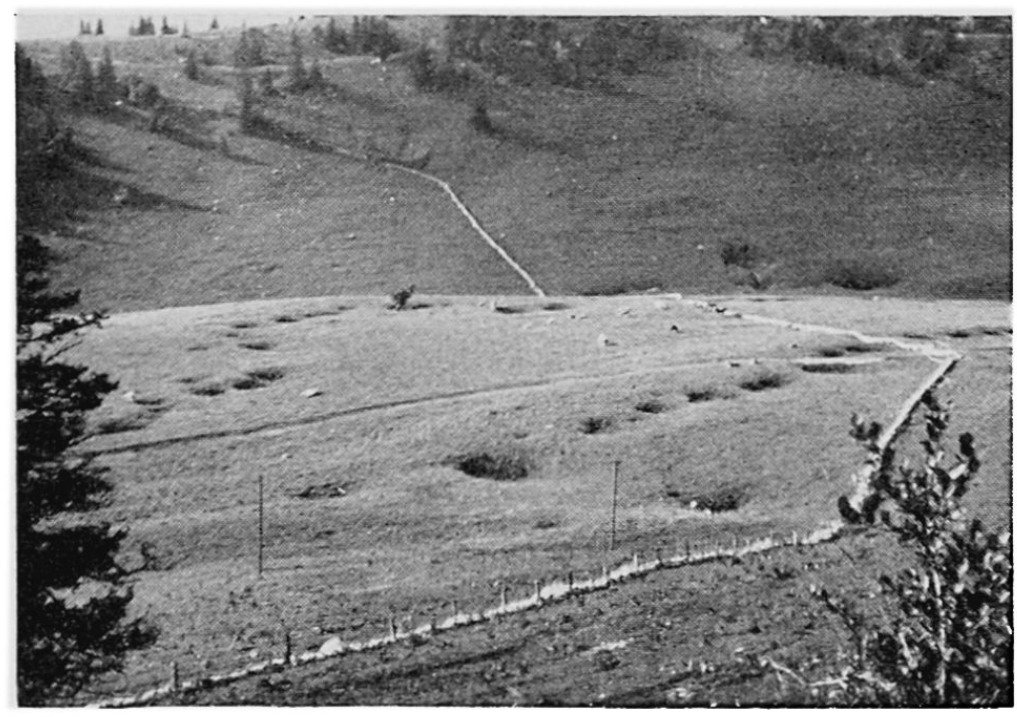

die Ackerbauzone schiebt sich weiter ins Beckeninnere vor; Versumpfung oder Dolinen sind heute ohne wesentliche landschaftliche Bedeutung.

Ein vergleichender Rundblick von der Mont Racine-Kette zeigt eindrücklich verschiedene Landschaftstypen des Neuenburger Jura: Im E das tiefere, klimatisch günstigere Val de Ruz, vom Seyon entwässert, ein buntes Mosaik intensiver Ackerbewirtschaftung, mit teilweise industriereichen Haufendörfern am Beckenrand und im Innern; darüber der ergänzende Weidebereich der Bergschulter - und im $W$ die höher gelegene Mulde von Les Ponts, ohne oberflächlichen Abfluß, mit Hochmoorresten, Torfstich, sauren Feuchtwiesen, mit spärlichen, spät reifenden Kornfeldern und dünnen Siedlungszeilen an den Talbodenrändern. So wird im überschauenden Vergleich die mittelbare Bedeutung der Verkarstung für die Jura-Landschaft, ihre physiognomische Karst-Intensität, sehr deutlich.

Das heikle Problem der sogenannten «aufgeschlossenen Poljen» mag wenigstens angedeutet werden. Danach sollen früher verkarstete, oberirdisch abflußlose Becken regressiv angezapft und der oberflächlichen Entwässerung tributär werden. MAcHATSchek (1905, S. 139 f.) und wohl auf seinen Spuren auch Schwabe (1939, S. 119) erwähnen als besonders schönes Beispiel das Val de Ruz, dessen mit glazialen Lehmen abgedichtetes Talbecken von einem verkarsteten Berggürtel rings umgeben wird. Der Hinweis MachatscheKs auf quartäre Karstsee-Ablagerungen findet jedoch in der geologischen Arbeit von FREI (1925) keine Bestätigung. FREI betrachtet sicher zutreffend die Seyon-Schlucht als tektonisch angelegt und, ähnlich wie bei einem Teil der Areuse-Schlucht, als mindestens gleichaltrig mit der Ketten-Faltung. Gutersohn (1958, S. 58) im Gegenteil glaubt, daß im Val de Ruz das Verhältnis von oberirdischem zu unterirdisch abfließendem Wasser heute zu Ungunsten des Seyon verschoben wird; einstige hangabdichtende Glaziallehne wurde heruntergeschwemmt, dadurch das Verkarstungsgebiet vergrößert. Diese Auffassung erscheint auch aus der allgemeinen Sicht der relativ jugendlichen Verkarstung im Jura als weit wahrscheinlicher.

\section{Höhlenbildungen}

Im Neuenburger Jura finden sich keine größeren Karsthöhlen, die etwa mit Tropfsteingebilden bedeutende Anziehungskraft hätten. Die bekanntesten liegen an der Areuse, wo die Cotencher-Grotte einige Funde der Moustérien-Zeit geliefert hat. Sie verdankt, wie die meisten Höhlen des Gebietes, ihre Entstehung einem Zusammenwirken von tektonisch-destruktiven und erosiv-lösenden Faktoren. Weitere Höhlen werden aus der Umgebung von Môtiers und auch von Les Verrières genannt, häufig wird auch die «grotte aux fées» in der Talkluse der Buttes erwähnt. Nirgends besteht indes eine direkte oder indirekte landschaftliche Wirkung solcher Höhlen. 


\section{Anthropogeographische Bemerkungen}

In der Art und Weise der menschlichen Ausnützung des je selteneren desto geschätzteren Wassers findet der jurassische Karst indirekt noch in anderer Weise seinen landschaftlichen Ausdruck.

Im allgemeinen bedeutet das natürlich eine bevorzugte Besiedlung wasserreicherer Gebiete. So verdankte Le Locle seine zuerst raschere Entwicklung dem reichlicher als um La-Chaux-de-Fonds vorhandenen Wasser. Am deutlichsten wird der landschaftliche Ausdruck dort, wo viel Wasser, verbunden mit starkem Gefälle, vorhanden ist, d. h. bei den ergiebigen Stromquellen. Ihre Lagen werden oft von dem Wechsel undurchläßiger und durchläßiger Schichten beeinflußt, lassen sich aber sonst keineswegs generell tektonisch fixieren. Hier konnten sich nach dem Quellaustritt eigentliche «Industriezeilen» bilden (Mühlen, Sägereien, Schmieden, Schleifereien usw.).

Größte Karstquellen des Gebietes sind die

$\begin{array}{lrl}\text { der Areuse bei St. Sulpice } & (500-20000 \mathrm{l} / \mathrm{sec} ., & \text { Mittel um } 4000 \mathrm{l} / \mathrm{sec} .) \\ \text { der Noiraigue } & (500-5000 \mathrm{l} / \mathrm{sec} ., & \text { Mittel um } 1500 \mathrm{l} / \mathrm{sec} .) \\ \text { der Serrière bei Neuchâtel } & (250-10000 \mathrm{l} / \mathrm{sec} ., \text { Mittel um } 2000 \mathrm{l} / \mathrm{sec} .)\end{array}$

Das Val de Travers ist besonders reich an Karstquellen, die auf die unterirdische Entwässerung der umgebenden Bergrücken hinweisen; neben den genannten finden sich weitere bei Fleurier und südlich von Môtiers, ganz abgesehen von den vielen, in der Areuse-Schlucht austretenden Wassern. Aber auch kleinere Gewässer wurden früher intensiv genutzt, besonders bei geringeren oder größeren Gefällstufen, oft gerade vor ihrem Versickern in einem Schlundloch.

All diese handwerklichen Betriebe (viele gehen bis aufs 16. Jahrhundert oder noch weiter zurück), seien es eigentliche «Industriezeilen» an Karstquellen oder Einzelbetriebe, hatten große Schwierigkeiten, sich gleichmäßig bis in die heutige Zeit weiterzuentwickeln: Die meisten erlagen ihnen im Rahmen der modernen industriellen Entwicklung im letzten Jahrhundert. Heute sind nur wenige alte Sägereien, aber z. B. keine einzige alte Mühle mehr im Betrieb; nirgends dient die ursprünglich gebrauchte Wasserkraft noch zum Antrieb. Das heißt, es bleibt höchstens der alte Betriebsstandort; die seinerzeit dafür ausschlaggebende Energie hingegen wird heute durchwegs anders - motorisch oder elektrisch - erzeugt. Über Fortbestehen oder Eingehen eines Kleinbetriebes entscheiden die Verkehrslage und die allgemeine wirtschaftliche Entwicklung. Solchen Faktoren fielen all die kleinen Getreidemühlen zum Onfer, ebenso abgelegene Schmieden und Walzwerke. An den alten Standorten blieben nur noch kleinere Sägereien erhalten (z. B. bei La Brévine, Le Gigot - Abb. 4) : Sie können von den Waldreserven des Jura zehren und genügen dem lokalen Bedarf.

Am Jura-Südfuß läßt sich nur im Falle von Serrières von einer sekundären Karstwirkung auf die heutige Landschaft sprechen. Dieses Industriegebiet konnte sich dank der günstigen Verkehrslage und unmittelbaren städtischen Nachbarschaft bis heute ständig fortentwickeln, wenn auch unter mannigfachem Wechsel des Produktionszieles und zunehmender Konzentration. Haвicht (1922) macht deutlich, wie die vielen Einzelbetriebe sich ständig der aktuellen Nachfrage anzupassen suchten, aber auch, wie sie im letzten Jahrhundert immer mehr zu wenigen großen Unternehmen verschmolzen.

ZsсноккE (1858, S. 267) zeichnet das ehemalige Serrières wie folgt: «Serrières, ist ein kleines Dorf, welches mehr malerisch-schön als bequem im Abgrund eines schmalen Felsenschlundes liegt. Es ist ein Dorf, fast nur aus Drahtzügen, Eisen- und Kupferhammerwerken, Papiermühlen und anderen Fabriken zusammengesetzt, die von einer nahen, reich hervorstürzenden Quelle betrieben werden. Der Raum ist für sie so eng, daß man in die Felsen Gewölbe einhauen mußte, um darin Mühlwerke, Magazine und Werkstätten einzurichten.»

Das heutige Serrières wird beherrscht von den modernen Fabriken der Suchard-Betriebe und einer Papierwarenfabrik.

Auch das Val de Travers, die alte Verbindungslinie des «vallis transversa» in 

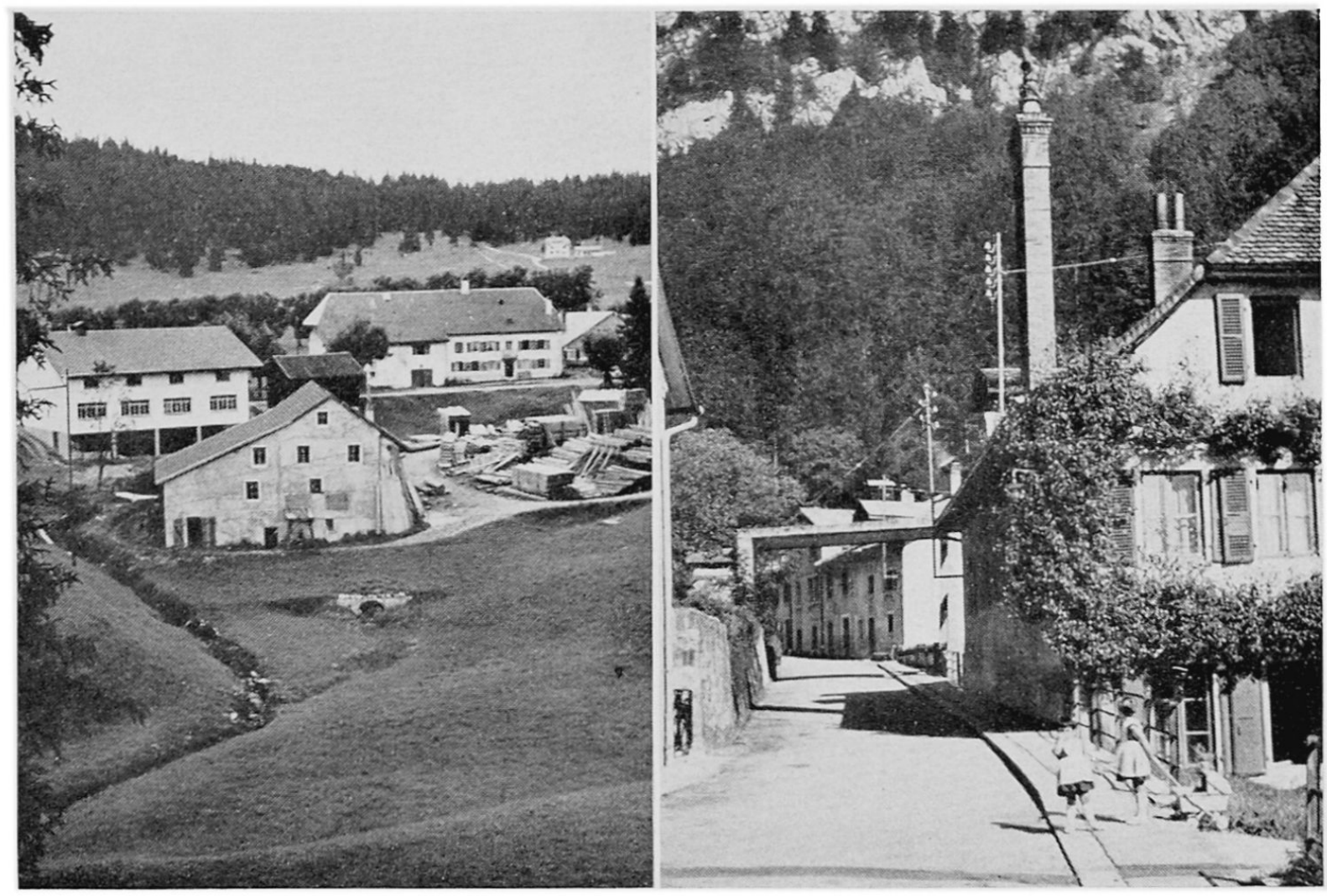

Abb. 4 Sägerei von La Brévine mit ehemaligem Wasserkanal. Abb. 5 (rechts) Ehemalige Industriezeile am obersten Lauf der Areuse bei St. Sulpice

Photo Christ

burgundische Lande, mit seinen häufigen Karstquellen zeigt industrielle Karst-Folgeerscheinungen: typische «Industriezeilen» in St. Sulpice und Noiraigue. In beiden Orten sind allerdings die ältesten Anlagen längst still gelegt. Ihre Gebäulichkeiten dienen höchstens noch als Wohnungen (Abb. 5). Neue Industrieen entstanden, von der Quelle abgerückt, unabhängig von der einst antreibenden Wasserkraft: die Portlandzementfabriken von St. Sulpice und Le Furcil, die unterdessen aus trust-politischen Gründen wieder still gelegt wurden, und eine Papierfabrik in St. Sulpice. Die Gefällsstufe der Areuse-Schlucht gibt in ihren wegsameren Teilen Gelegenheit, die Areuse oder die aus den Hängen zahlreich austretenden Quellen auszunützen, sei es zur Wasserversorgung oder zur Elektrizitätsgewinnung. Champ du Moulin bezeichnet einen alten, nach Gutersohn auf das 14. Jahrhundert zurückgehenden Betriebsstandort: Heute, vom Verkehr abgeschnitten, ist er höchstens noch Ausflugsort. Ähnlich liegt «La Roche» nun abseits des Verkehrs (nordwestlich von Couvet) in der Höhe: ehemals eine in Fels eingehauene Schmiede. Daß auch der Doubs früher von vielen, die Wasserkraft von Fluß und zuströmenden Karstquellen nutzenden Betrieben gesäumt war, beweisen noch einzelne Namen und Mauerreste (Moulinet bei Les Brenets, La Forge, Moulin Robert). Heute wird lediglich das bei Le Châtelot aufgestaute Doubswasser zur Erzeugung elektrischer Energie verwendet. Das Becken von Le Locle, früher im westlichen Teil meist versumpft, ist jetzt durch den Felsdurchstich von Col des Roches, in den der drainierte «Bied» seit 1805 abgeleitet wird, landwirtschaftlich weitgehend nutzbar gemacht. Bekannt waren die unterirdischen Mühlen, die der versickernde Bied bei Col des Roches getrieben hat.

Zsснокке (1858, S. 274) berichtet davon: «Im Mühlgebäude selber führt vom obern Mühlenwerk eine in Fels gemeißelte Treppe wie in einen Keller hinab. Aber der Keller erweitert sich. In der Finsternis drunten arbeitet eine zweite, weit tiefer noch eine dritte und noch tiefer eine vierte Mühle. Dasselbe Wasser, welches die obern Werke und Räder treibt, stürzt auf die untersten, wo dann ein Felsenrachen, den man in der Landessprache Chaudière nennt, zuletzt 
alle Fluten verschluckt. Das Stampfen, Rollen und Lärmen der Mühlen in diesem Abgrund über und unter uns, welche die vorhängenden Klippen zu zermalmen drohen, das Durcheinandersausen, Pfeifen und Donnern der herabfahrenden Wogen, die sich zwischen den finstern Labyrinthen des Gesteins tausendfach zerschlagen, der trübe Schimmer der Lampen, ihr unheimlicher Widerglanz von den feuchten Umgebungen, ... wo sich Alles, Flut und Fels, durcheinander zu bewegen scheint - es ist wie ein Vorhof der Hölle, worin, statt der Feuerflammen, tanzende Wassersäulen gaukeln und rauschen.»

Gegenwärtig steht an dieser Stelle der große Grenz-Schlachthof mit seinen Gefrier- und Lagerhäusern.

Auch die Ronde, ein Grundwasseraufstoß, auf dem Stadtgebiet von La Chaux-deFonds entspringend und heute mit für die städtische Wasserversorgung gefaßt, betrieb einst eine in den Fels eingehauene Mühle am Eingang zur Valanvron-Schlucht. Ähnlich wurde auch das spärliche Abflußwasser der Crosette-Talung vor der Versickerung aufgestaut und genützt.

Fig. II gibt eine Zusammenfassung der früheren Betriebsstandorte, der an alten Standorten gebliebenen Betriebe und der heutigen Industrie-Verbreitungsgebiete. Die Konzentration auf wenige Hauptgebiete (und -Industrien), ist deutlich gegenüber der früheren Streuung der verschiedenartigsten Betriebe.

Die Bevölkerung des Jura, vor allem die bäuerliche, ist oft auch heute noch außerordentlich anspruchslos; viele Einzelsiedlungen leben nur von Zisternenwasser. Die moderne Technik mit Pumpstationen, Großreservoiren und ausgedehnten Leitungsnetzen, die sorgfältige Suche und Fassung aller vorhandenen Grundwasseraufstöße lassen aber gerade in den größeren Orten die Nachteile eines wasserarmen Karstgebietes völlig vergessen. Immerhin: Allein auf dem weiteren Stadtgebiet von La Chauxde-Fonds sind mindestens $750 \mathrm{Zisternen} \mathrm{-} \mathrm{heute} \mathrm{meist} \mathrm{ungebraucht} \mathrm{-} \mathrm{bekannt!} \mathrm{Die}$ großzügige Wasserversorgung aus den Quellen der Areuse-Schlucht über den Solmont genügt gut für einen täglichen Wasserbedarf von über $250 \mathrm{l}$ pro Einwohner dieser Stadt. (Vgl. dazu auch die Wasserversorgung der Freiberge aus dem Val St. Imier über den Mont Soleil.)

\section{Zusammenfassung}

Die naturlandschaftliche Bedeutung der Karstformen im Jura ist gering; es handelt sich um relativ jugendliche Verkarstung. Снавот (1927, S. $324 \mathrm{ff}$.) weist dies mit überzeugenden Argumenten nach, indem er die Entwicklungsformen bei oberirdischer und bei Karst-Erosion miteinander vergleicht: Die Karst-Erosion geht ungleich langsamer, bildet nur wenig ausgeprägte Formen. Überall im Jura hingegen ist ein Gebiet oberirdischer Entwässerung und Erosion nahe: Tektonische Anlage und Oberflächen-Entwässerung haben den heutigen Jura entscheidend geprägt. Lokale Peneplains im Sinne von HetTner (1912), als Karstverebnungen mit jeweils unterschiedlicher Erosionsbasis, spielen keine Rolle im heutigen Relief.

Große Reliefenergie befördert die oberirdische Entwässerung: Dies gilt besonders für die steilen Schenkel des Kettenjura. Bevorzugte Verkarstungsgebiete liegen auf flachen Antiklinalplateaux, in denen bereits durch die Faltung (Zerrung im Faltenscheitel) Spalten und Klüfte vorhanden sind, die das Wasser zur unterirdischen Zirkulation aufnehmen. Gemäß der vom Jura-Südfuß nach Norden abnehmenden Reliefenergie findet sich im Neuenburger Jura der typische Gegensatz der oberflächlich entwässerten Vallées und der abflußlosen Hochmulden; die verkarsteten Berggebiete werden gegen $\mathrm{N}$ breiter. Der Untergrund wirkt entscheidend bei der hydrographischen Ausgestaltung einer Landschaft mit. In unserem Gebiet werden die weiten Talungen durch Molassesedimente oder glaziale Deponien abgedichtet, die abflußlosen Hochmulden vermooren dadurch mehr oder weniger. Untergeordnet treten auch auf Antiklinalen und in Comben dichtende jurassische Tone oder Mergel an die Oberfläche.

Danach läßt sich generell der Talkarst mit randlichen Dolinen, Ponoren und Moorbildung auf undurchläßiger Unterlage, und der physiognomisch weniger intensive Bergkarst auf kalkigem Untergrund - höchstens mit kleinräumigen Hochmooren 


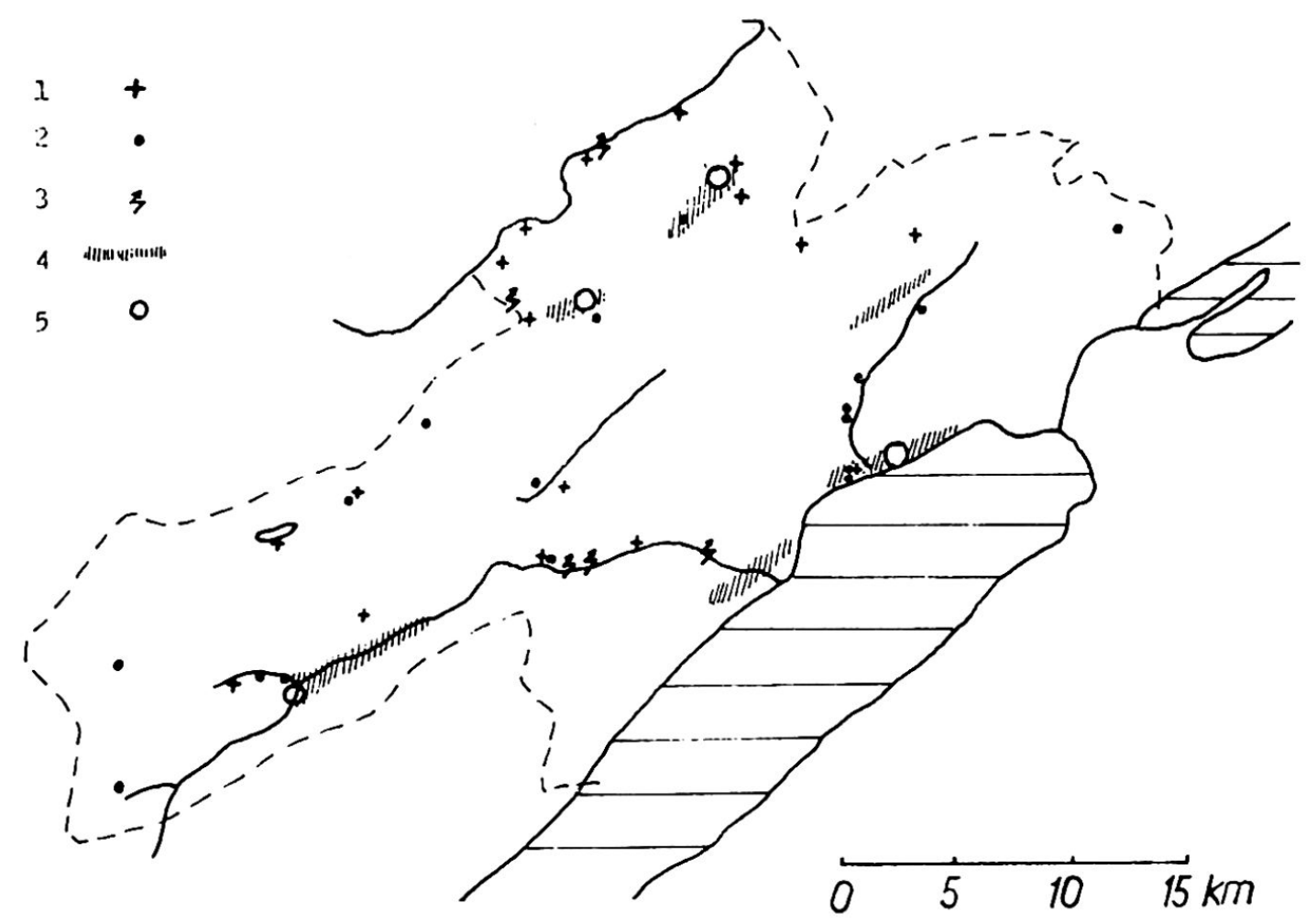

Fig. II Betriebstandorte und Industrie. 1 Frühere Betriebe mit Wasserkraft, heute verschwunden; 2 Heutige Betriebe an ursprünglichem Standort, nur noch Sägereien; 3 Elektrizitätswerk, WasserPumpwerk; 4 Wichtigste Industriegebiete der Gegenwart; 5 Ortschaften

in Antiklinal-Aufbrüchen - unterscheiden. Dolinen sind auch in unserem Gebiet die häufigste Karstform; auch hier gilt der Satz von Cнавот (1927, S. 324) : «Si nombreuses que soient les dolines, elles n'altèrent pas notablement le caractère normal de la topographie initiale». Dolinenreihen längs stratigraphischen Grenzen (Kalk-MergelWechsel) sind häufig, seltener an tektonischen Störungen. Ihre landschaftliche Intensität im Zusammenhang mit der jeweiligen Umgebung ist nach Form, Größe, Bodenbedeckung, Tal- oder Berglage verschieden.

Ponore spielen nur mittelbar eine Rolle als lokale Erosionsbasis eines geschlossenen Beckens. Jurassische «bassins fermés» sind nach Hydrographie, Morphologie und Funktion nicht völlig den dinarischen «Poljen» gleichzusetzen. Oberflächlich fließendes Wasser in einer verkarsteten Gegend ist ein wesentlicher anthropogeographischer Faktor. Danach kann sich Besiedlung und Verkehrsentwicklung orientieren. In unserem Gebiet läßt sich die beschränkte anthropogeographische Bedeutung vor allem in der Lage von Gewerbebetrieben erkennen und in deren zeitlichem Wandel verfolgen. Mühlen, Schmieden und andere handwerkliche Betriebe nutzten einst die Wasserkraft. Alte Betriebsstandorte bleiben bis heute umso eher erhalten, je bessere heutige Verkehrslage sie aufweisen. Dabei verändern die Betriebe teilweise mehrfach, im Rahmen der Entwicklung zur modernen Fabrikindustrie, ihr Produktionsziel, oder aber sie bleiben auf lokale Bedürfnisversorgung beschränkt (Sägereien). Die heutige Verkehrslage wird generell vom Jura-Südfuß gegen $\mathrm{N}$, ins Jura-Innere und gegen die Landesgrenze zu, schlechter.

Die wechselnde Bedeutung der Karstformen läßt sich folgendermaßen zusammenfassen. Für abgelegene Hochmulden, z. Tl. mit Moor, und breite Gewölberücken, mit Wald und Weide - gilt: Karstformen in der Naturlandschaft sind häufig und je nach der Umgebung und Ausbildung von beschränkter Bedeutung. Die heutige Verkehrslage jedoch ist generell schlecht, so daß die mittelbaren, anthropogenen Auswirkungen zurücktreten, alte Betriebsstandorte überwiegend eingehen. 
Für zum Vorland entwässernde Muldentäler, und den vorwiegend von Reben bestandenen Jurafuß, gilt: Karstformen sind sehr selten, mit Ausnahme der häufigen Stromquellen, die je nach ihren Schüttungsmengen typische Industriezeilen hervorrufen. Die heutige bessere Verkehrslage trägt dazu bei, daß die sekundären, anthropogenen Auswirkungen des Karstes bei weitem die naturlandschaftlichen überwiegen.

\section{LITERATUR}

BeLt., E.: Monographie de la vallée du Doubs entre Les Brenets et Biaufond. Bull. Soc. Neuch. Géogr. XL, 1931, S. 5 - 133 - Birot, P.: Problèmes de Morpholozie karstique. Ann. de Géogr. LXIII, 1954, S. 161-192 - Boegli, A.: Probleme der Karrenbildung. Geographica Helvetica 6,1951, S. 191-204 - Снавот, G.: Les plateaux du Jura central. Diss. Strashourg 1927 - EPPl.E, P.: Geologische Beschreibung der Umgebung von Sonceboz im Berner Jura. Diss. Bern 1946 - Erzinger, E.: Die Oberflächenformen der Ajoie. Diss. Basel 1943 - FAVRE, J.: Description géologique des environs du Locle et de la Chaux-de-Fonds. Ecl. Geol. Helv. 11/1911, S. 369-475 - FrEI, E.: Zur Geologie des südöstlichen Neuenburger Jura, insbesondere des Gebietes zwischen Gorges de l'Areuse und Gorges du Seyon. Beitr. Geol. Karte, N. F. 55. Lfg., Bern 1925 - FrueH, J.: Geographie der Schweiz, 3 Bde., bes. 3. Bd., St. Gallen 1938 - Grund, A.: Der geographische Zyklus im Karst. Zschr. Ges. Erdkunde Berlin 1914 - Gutersohn, H.: Landschaften der Schweiz. Zürich 1950 - Gutersohn, H.: Geographie der Schweiz, Bd. I: Jura. Bern 1958 - НАвіCHт, W.: Les industries de Serrières à travers les âges. Bull. Soc. Neuch. Géogr. XXXI'1922, S. 5-127 - Heln, Alb.: Geologie der Schweiz; Bd. 1: Molasseland und Juragebirge. Leipzig 1919 - Hettrer, A.: A us dem Schweizer Jura. Geogr. Zschr. 18,1912, S. 515-521 - JaCot-Guil.laRmod, Ch.: Carte des bassins fermés du Jura Suisse. in: E. de Margerie: Le Jura, Ière partie, Bibliographie sommaire du Jura français et suisse. Paris 1922 (Karstkommission:) Das Karstphänomen in den verschiedenen Klimazonen. Bericht v. d. Arbeitstagung der internat. Karstkommission. Erdkunde 7/1954, S. 112-139 - LenmanN, O.: Die Hydrographie des Karstes. Wien und Leipzig 1932 - Louls, H.: Die Entstehung der Poljen und ihre Stellung in der Karstabtragung, auf Grund von Beobachtungen im Taurus. Erdkunde 10,1956, S. 33-53 - Maсhatscheк, F.: Der Schweizer Jura. Versuch einer geomorphologischen Monographie. Erg. Heft 150 zu Petermanns Mittlg. Gotha 1905 - MachatsCheK, F.: Geomorphologie. 6. Aufl. Stuttgart 1954 - Mühi.eThaler, Ch.: Etude géologique de la région des Verrières. Bull. Soc. Neuch. Sc. nat. 56,1931 S. 121-300 - RiCKENBACH, E.: Description géologique du Val-de-Travers entre Fleurier et Travers, du Cirque de Saint-Sulpice et de la Vallée de la Brévine. Bull. Soc. Neuch. Sc. nat. 50,1925, S. 1-76 - (Schaefer, I.:) Artikel "Karst", im Fischer-Lexikon Geographie, hrsg. v. FochLER-HaUke. Frankfurt 1959 - SChaRdt, H.: Mélanges géologiques sur le Jura neuchâtelois et les régions limitrophes (Origine de la source de l'A reuse). Bull. Soc. Neuch.Sc. nat. 32, 1903-04, S. 81-139 SCHARDT, H.: Mélanges géologiques .... (cours des eaux de la Ronde) Bull. Soc. Neuch. Sc. nat. 37, 1909-10, S. 364-79 - SCHARDT, H. und DuBors, A.: Description géologique de la région des Gorges de l'Areuse. Bull. Soc. Neuch. Sc. nat. 30,1901-02, s. 195-352 - SchwabE, E.: Morphologie der Freiberge. Diss. Basel 1939 - SUTER, H.: Geologische Untersuchungen in der Umgebung von Les Convers Vue des Alpes. Diss. Zürich 1920 - ThiéBaud, Ch.-E.: Etude géologique de la région Travers, Creux du Van, Saint-Aubin. Bull. Soc. Neuch. Géogr. XLV, 1937, S. 5-74 - Vosseler, P.: Die Landschaften der Schweiz (BI. 5: La Chaux-de-Fonds). Bern 1929 - ZsсноккE, H.: Die Schweiz in ihren klassischen Stellen und Hauptorten. St. Gallen 1858.

\section{K A RTEN}

Landeskarte der Schweiz $1: 25$ 000. Blätter 1164 (Neuchâtel), 1163 (Travers), 1162 (Les Verrières), 1144 (Val de Ruz), 1143 (Le Locle) - Landeskarte der Schweiz 1:50 000, Blatt 232 (Vallon de St. Imier) - Jurakarte 1:50 000, Blätter II und VI - Geolog. Generalkarte der Schweiz 1:200000, Blatt 1 (Neuchâtel) - Geotechn. Karte der Schweiz 1:200 000, m. Erl., Blatt 1.

\section{KARSTIFICATION DANS LE JURA NEUCHÂTELOIS}

L'étude traite d'abord de l'effet des formations karstiques sur le paysage dans le canton de Neuchâtel. En général, leur importance est peu considérable. La vigueur du relief et le soussol sont les conditions déterminantes pour le développement hydrographique d'un paysage; en conséquence, on peut distinguer des formations karstiques de montagne et de vallée.

Les lapiés, les dolines, les bassins fermés sont décrits selon leur importance et rattachés aux paysages respectifs. Enfin, l'étude porte sur l'influence des eaux courantes de surface, en particulier des sources vauclusiennes, sur la géographie humaine d'une région karstifiée, par exemple sur la localisation des établissements artisanaux ou des rubans d'industrie. 